\title{
COVID-19 antibodies on trial
}

\author{
With the first readouts of trials of antibodies against COVID-19 appearing and others coming thick and fast, \\ Nature Biotechnology asked a group of experts to comment on the challenges and timelines for these products.
}

Linda J. Saif is at the Ohio Agricultural Research and Development Center at The Ohio State University College of Veterinary Medicine in Wooster, Ohio, USA.

Dennis Burton is at the Scripps Research Institute in La Jolla, California, USA.

Erica Ollmann Saphire is at the La Jolla Institute for Immunology, La Jolla, California, USA. George Scangos is at Vir Biotechnology in San Francisco, California, USA.

George Georgiou is at the University of Texas in Austin, Texas, USA.

Tillman Gerngross is at Dartmouth College in Hanover, New Hampshire, USA. Jake Glanville is at Distributed Bio in South San Francisco, California, USA.

In October, US President Donald Trump received Regeneron Pharmaceuticals' experimental monoclonal antibody (mAb) cocktail REGN-COV2 as part of his treatment for COVID-19. Buoyed by a positive response, both Regeneron and Eli Lilly have filed requests for Emergency Use Authorization from the US Food and Drug Administration, although Lilly had to pause clinical testing because the trial crossed a predetermined safety threshold. Lilly's product, LY-CoV555, is a human IgG1 $\mathrm{mAb}$ targeting the spike $(\mathrm{S})$ glycoprotein. These and 11 other experimental $\mathrm{mAb}$ treatments targeting the SARS-CoV-2 S protein are undergoing human testing (Table 1), with at least another 150 other antibodies in discovery research. Neutralizing mAbs promise an adjunct to vaccines and traditional drugs in the treatment of COVID-19. Here, a group of experts comments on the state of the art in antiviral $\mathrm{mAb}$ discovery and development, and the challenges ahead.

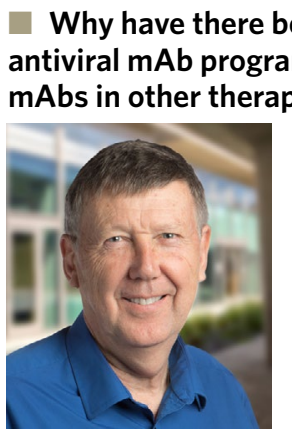

Dennis Burton, Scripps Research Institute.

might still be required if, for example, an aged population or immunocompromised individuals do not respond to it. For antiviral therapy, expectations have been relatively low, but the discovery of potent broadly neutralizing antibodies to highly antigenically variable viruses like HIV and influenza and the demonstrated efficacy of even a single $\mathrm{mAb}$ against established Ebola virus infection in humans are changing all that. Also, note that many viral infections do not represent large markets, are not attractive to commercial concerns and may need government or philanthropic support for the development of mAbs.

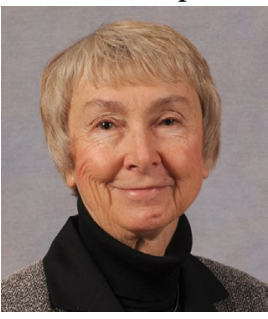

Linda J. Saif, The Ohio State University. Credit: Ken Chamberlain
Linda J. Saif: It takes a major investment of time and funding to develop and test functional human antiviral mAbs. Compared with therapeutic mAbs for other indications, the bar of finding molecules effective at neutralizing virus is a high one. For example, in COVID-19, the screening of memory B cells from recovered cases finds many mAbs that bind to SARS-CoV-2 S protein and its receptor-binding domain (RBD), but neutralizing $\mathrm{mAbs}$ are of lower frequency. Once neutralizing $\mathrm{mAbs}$ have been found, they need to be further characterized in vitro for stability, epitope specificity and binding, and cross-competition, and in structural/ functional studies. Next, those with the highest neutralizing potency in vitro are tested therapeutically and prophylactically in dose-response studies in SARS-CoV2-challenged animal models. Finally, only a few highly potent and efficacious mAbs are selected to advance to human clinical trials. George Georgiou: The success rate in the development and clinical use of anti-infective antibodies in general has been modest. The reasons for that are complex, but if one were to make a generalization, then the principal factors would be because of a combination of moderate efficacy and cost/reimbursement considerations. For example, even for Synagis, which has been commercially and clinically successful for prophylaxis to RSV infection in high-risk infants, efficacy in preventing hospitalizations is of the order of $60 \%$. In influenza, multiple broadly neutralizing antibodies have entered clinical development, but so far the reported efficacy has been rather underwhelming. Nonetheless, progress in the field of antibody discovery technologies, better understanding the mode of action of anti-infective antibodies and much more cost-efficient manufacturing technologies suggest that a new generation of more effective and relatively affordable $\mathrm{mAb}$ therapeutics for viral diseases is increasingly likely.

Jake Glanville: Antiviral antibody therapies don't get enough credit. In addition to RSV (Synagis) and Ebola (REGN-EB3 and others), several other success cases of antiviral antibodies should be mentioned: rabies (HYPERRab), HIV (Trogazo), anthrax (raxibacumab), hepatitis $\mathrm{C}$ (bavituximab) and a dizzying portfolio of military research into $\mathrm{mAbs}$ for rare but exceedingly deadly viruses. Antibody therapies in the form of polyclonal convalescent sera have been used for over a century, including for 1918 Spanish flu, smallpox (vaccinia immunoglobulin), measles, Bolivian hemorrhagic fever, Argentine hemorrhagic fever, Ebola and Lassa hemorrhagic fevers, cytomegalovirus, hepatitis B virus, vaccinia virus, varicella zoster virus, RSV and West Nile virus.

That said, it is certainly the case that the $\mathrm{mAb}$ therapy drug sector is dominated by oncology and immune targets. A likely reason for this is that these are high-value sectors where antibodies have proven very effective and can command a premium in a limited number of patients. Monoclonal therapies don't scale particularly well due to virus (RSV) and Synagis, an mAb approved in 1998). Even if there is a vaccine, mAbs 
historical reliance on Chinese hamster ovary (CHO) cell manufacturing, and therefore high-value (tens of thousands of dollars per infusion per patient), high-impact markets are preferred. This manufacturing and pricing model is not well aligned with infectious disease, where very large numbers of doses may be required and are expected/ need to be relatively affordable due to community expectations around costs of antivirals and antibiotics.

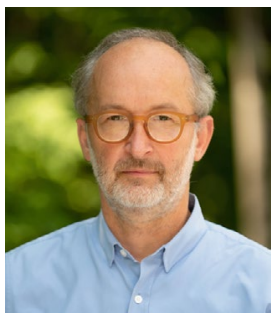

Tillman Gerngross, Dartmouth College. Credit: Genevieve de Manio

Tillman Gerngross: $\mathrm{mAb}$ prophylaxis is generally considered for viruses that have proven refractory to vaccine development (for example, HIV and RSV) or for populations that do not mount strong immune responses to vaccination (for example, infants, elderly and immunocompromised individuals). As an example, the RSV mAb Synagis has been successfully used for the prevention of severe RSV disease for decades in infants at high risk. For therapy, mAbs have historically shown limited success, but this is changing with the discovery of antibodies with remarkable neutralization breadth and potency. Over the past few years, potent neutralizing antibodies to Ebola and HIV have shown therapeutic efficacy in humans.

Unfortunately, one of the reasons why there have been very few successful antiviral mAbs programs compared with $\mathrm{mAbs}$ in other therapeutic areas (for example, cancer and autoimmunity) is because many viral diseases are episodic, acute and primarily impact people in developing countries, making them commercially unattractive for biotech and pharma companies. Governments and non-profit agencies will need to invest in the development of mAbs for viral diseases to change this paradigm.

\section{What aspects of the polyclonal} antibody-mediated response to coronaviruses in patients have informed $\mathrm{mAb}$ engineering and design for COVID-19? L.J.S.: Knowledge that antibodies to $S$ protein and the RBD induced in patient sera can neutralize SARS-CoV-2 infectivity in vitro and the timeline and isotypes of serum antibody responses induced have informed $\mathrm{mAb}$ design. However, knowledge is more limited on IgA antibody responses to SARS-CoV-2 in mucosal secretions or tissues. There is also limited information on the timeline of antibody responses in serum to each of the SARS-CoV-2 open reading frame proteins and if antibodies aside from those that bind to the $S$ protein play a role in protection. Convalescent plasma with adequate but undefined virus-neutralizing antibody titers may aid in recovery if given early in the course of disease, based on ongoing observational studies, but controlled clinical trials are needed to confirm safety and efficacy.

G.G.: The data with convalescent plasma therapy argue that mAbs are likely to be beneficial in the management of severe disease. I suspect that $\mathrm{mAbs}$ may prove to be particularly critical as a prophylactic strategy for high-risk groups - for example, people who are immunocompromised and possibly the elderly, who have suboptimal responses to vaccination against respiratory pathogens. Passive immunization with mAbs will almost certainly require the use of Fc-engineered antibodies having amino acid substitutions that greatly prolong the persistence of antibodies in circulation from $\sim 21$ days to $\sim 85$ days.

The development of SARS-CoV-2 neutralizing $\mathrm{mAbs}$ has been driven by the premise that higher neutralization potency should translate to greater therapeutic benefit. This makes perfect sense. I should note that several studies have found higher neutralization titers in patients with more severe disease; however, this paradox may well be a consequence of the kinetics of humoral response and inflammation status, rather than evidence that higher levels of neutralizing antibodies fail to resolve the disease.

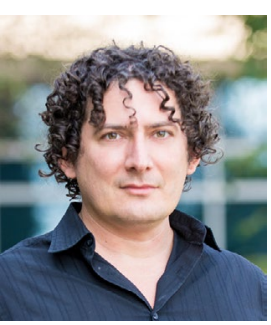

J.G.: Polyclonal responses provide an early window to ask whether antibodies can be protective against the novel coronavirus.

Although convalescent

Jake Glanville, Distributed Bio.

Credit: JCo Studios

plasma studies are confounded by great variation in quantity and quality of neutralizing responses in recovered donors, retrospective studies at Mount Sinai Medical Center [New York] have shown dose-dependent and neutralization-level-dependent benefits that suggest that antibodies can work in humans to stop the coronavirus. We have seen polyclonal sera as well as mAbs protect as well as treat mice, hamsters and non-human primates (NHPs). All signs suggest that $\mathrm{mAb}$ therapies will provide a potent treatment option against the coronavirus: now it is a waiting game for human trials to complete.

Why are S protein and its RBD the sole focus for antiviral COVID-19 mAb programs in the clinic?

L.J.S.: The SARS-CoV-2 S protein and its RBD are the major target because neutralizing $\mathrm{mAbs}$ to them have been shown in vitro in cell culture to effectively inhibit virus binding to the host receptor, human angiotensin-converting enzyme (hACE2), and thus block viral entry into the cell. When administered in vivo, several of the high-potency neutralizing mAbs passively protect SARS-CoV-2-challenged animal models ${ }^{1-3}$. Several mAbs binding outside the RBD are also capable of virus neutralization in vitro through undetermined mechanisms ${ }^{4}$.

At this stage, little is known about the potential for SARS-CoV-2 mAbs to effectively block intracellular viral replication. A related interesting observation pertains to enteric rotaviruses. Neutralizing $\mathrm{mAbs}$ to either of the two outer surface attachment proteins, viral protein (VP) 4 and VP 7, but not mAbs to the inner capsid protein VP 6, can block virus replication in vitro. However, only variable heavy chain $\left(\mathrm{V}_{\mathrm{HH}}\right)$ llama nanobodies to conserved VP 6 epitopes could broadly neutralize homologous and heterologous rotavirus strains in vitro. In our studies, they also passively protected piglets from human rotavirus infection in vivo when administered orally at 36 hours pre-rotavirus challenge (through 9 days) ${ }^{5}$. The mechanism by which $\mathrm{V}_{\mathrm{HH}}$ nanobodies to rotavirus VP 6 inhibit rotavirus replication is unclear; they could potentially penetrate the rotavirus outer capsid, bind VP 6 and block decapsidation, inhibiting early steps of virus replication. Alternatively, anti-VP 6 nanobodies could block viral transcription at the start of the intracellular viral replication cycle due to their small size and ability to penetrate into the infected cells.

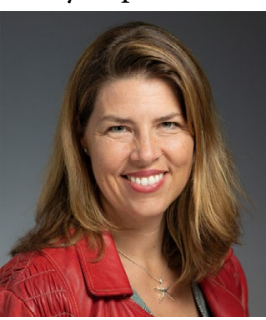

Erica Ollmann Saphire, La Jolla Institute for Immunology.
Erica Ollmann Saphire: Antibodies are most often used against proteins displayed on the outside of the virus or outside of the infected cell. By targeting S protein on the SARS-CoV-2 surface, antibody can neutralize infectivity of that virus. By targeting S protein on the surface of an infected cell, antibody can tag that cell for destruction by the immune system.

The other, non-spike viral targets are good targets for small-molecule 
inhibitors - protease inhibitors, for example. For other viruses, there are reports of some antibody formats that can be internalized into cells where they can exert their function. So an antibody might perhaps be able to target an internal protein, but they are generally developed against surface proteins.

\section{What do we know about the roles of different antibody isotypes and subtypes and their tissue localization in combating viral infection?}

L.J.S.: Different antibody isotypes and sub-isotypes have different functional activities and tissue locations, with secretory IgA dominant at mucosal surfaces. Previous work reported that two oligomeric IgA mAbs to rotavirus VP 6 did not show neutralizing activity in vitro ${ }^{6,7}$. When administered to mice in vivo in a backpack tumor model, they passively protected against infection of mice, presumably by inhibiting viral transcription. The authors suggested that protection in vivo was mediated by the binding of VP 6 oligomeric IgA $\mathrm{mAb}$ to rotavirus, which protects by intracellular neutralization during oligomeric secretory IgA transcytosis in the mouse gut. Thus oligomeric, but not monomeric, IgA or IgG mAbs could be transcytosed via the polymeric immunoglobulin receptor on target gut epithelial cells and protect against rotavirus intracellular infection in the intestine. Other pathways for intracellular virus neutralization by $\operatorname{IgA}$ antibodies have also been described ${ }^{8}$.

For animal coronavirus infections, it is known that locally produced IgA antibodies are associated with protection of mucosal tissues, including the upper respiratory tract and the gastrointestinal tract ${ }^{9}$. This is also documented for intranasal delivery of live-attenuated FluMist influenza vaccine, which induces $\operatorname{IgA}$ antibodies in the upper respiratory tract and protection, despite lower serum IgG antibodies. Circulating IgG antibodies are associated with systemic protection. As shown for intramuscular delivery of inactivated influenza vaccines, serum IgG antibodies to influenza are potentially effective in preventing lung infection and disease. It is also likely that inflammation induced after lung infection will increase the transudation of serum IgG antibodies or $\mathrm{mAbs}$ to the lung.

G.G.: For SARS-CoV-2, the most potent anti-S protein neutralizing antibodies an overwhelming number of which are directed to the RBD - can block infection in vitro at concentrations in the nanomolar range. So far, data from animal studies suggest that for protection pre-infection (or for mitigating lung damage post-infection) in animal models, even very potent antibodies need to be administered into the circulation at appreciable concentrations, typically in the range of $10 \mathrm{mg}$ per $\mathrm{kg}$ weight or higher. This may be because transcytosis of IgG into the lung epithelium is rather inefficient. The rule of thumb is that the concentration of antibodies in the lungs is 200-500 times lower than in circulation. What concentration of therapeutic antibody can be achieved in the upper respiratory tract and for how long is currently unknown.

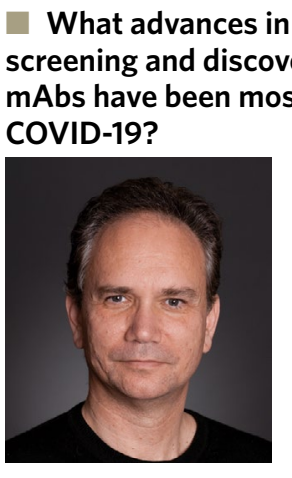

George Georgiou, University of Texas, Austin. Credit:

Marsha Miller neutralizing SARS-CoV-2 antibodies have been generated using practically every method of antibody discovery, from combinatorial libraries to animal immunization followed by humanization, reflecting the palette of technologies used for antibody technology in academia and industry. However, most well-characterized, highly potent neutralizing antibodies have come from single-cell cloning of patient peripheral B cells or from humanized mice. As a general rule, human antibodies often have better process 'developability' characteristics relative to antibodies from combinatorial libraries. An important consideration for therapeutic development is polyreactivity. Many of the best-characterized broadly neutralizing antibodies for viruses, such as HIV-1 or influenza, are polyreactive; in other words, they show binding to lipids and unrelated antigens. This can negatively impact half-life and biodistribution or, more worryingly, might elicit adverse effects. The extent to which SARS-CoV-2 neutralizing antibodies are polyreactive is not fully known. However, it is encouraging that a recent report by Pamela Bjorkman shows that at least certain classes of $\mathrm{mAbs}$ raised to S-protein epitopes are not polyreactive.

In vivo production of DNA- or RNA-encoded mAbs following gene therapy has been demonstrated in animal models. For SARS-CoV-2, a high concentration of circulating antibodies will be necessary, as discussed above, and I am skeptical as to whether such large amounts of protein can be produced endogenously in humans, especially without inducing excessive cellular stress and associated adverse effects. J.G.: The appearance of the novel coronavirus in 2020 acted as a global benchmark of the entire antibody discovery field in its golden age. The best approaches appeared to be those that used 'antibody repertoires' that were skewed toward success, rather than entirely naive systems. Screening B cells from convalescent patients appears to be an approach successfully used by multiple groups. This approach requires a lot of financial resources and technology to isolate and sequence single cells and then perform high-throughput screens to determine the neutralizing $\mathrm{mAbs}$, but has the clear advantage that 'first in human' was done up front. Likewise, transgenic mice are effective systems antibody generation platforms for similar reasons - they can be immunized and their serum tested along with the clones.

Our approach was to leverage years of research by identifying anti-SARS antibodies with known crystal structures, which are already known through years of research to be neutralizing and protective in vivo. These antibodies were then rapidly evolved to recognize the novel coronavirus while retaining their functions. The approach enabled us to spend much less time and resources in screening and focus immediately on optimizing (increasing affinity and thermostability for subcutaneous delivery). The benefit of this computationally guided approach is that engineering was completed by the beginning of April 2020, keeping pace with much better-funded efforts. This suggests that the future of antibody discovery and optimization will be in this direction. T.G.: Human B-cell cloning has proven to be the most successful approach to rapidly identifying potent neutralizing antibodies. However, nature may not necessarily provide the most optimal molecules. In our case, after initially isolating antibodies from a SARS survivor with rare breadth of neutralization across clade I sarbecoviruses, we wanted to further improve potency to SARS-CoV-2. As such, we believe that the combination of B-cell cloning followed by protein engineering (affinity) gave us the best molecules.

\section{What are the pros or cons of particular scaffold antibody formats in antiviral therapy?}

L.J.S.: Fully human mAbs have shown various levels of effectiveness in SARS-CoV-2challenged murine, hamster and NHP 
Table 1 | SARS-CoV-2 mAbs in clinical development

\begin{tabular}{|c|c|c|c|c|}
\hline Sponsor & Product & Clinical stage & Trial ID & Study \\
\hline Regeneron/NIAID & $\begin{array}{l}\text { REGN-COV2 (REGN10933 + } \\
\text { REGN10987; human IgG1 mAbs } \\
\text { targeting S protein epitope) }\end{array}$ & Phase $2 / 3$ & NCT04452318 & $\begin{array}{l}2,000 \text { healthy adults with infected } \\
\text { people in household }\end{array}$ \\
\hline Regeneron/NIAID & REGN-COV2 & Phase $2 / 3$ & NCT04426695 & $\begin{array}{l}2,970 \text { hospitalized adults with } \\
\text { COVID-19 }\end{array}$ \\
\hline Regeneron/NIAID & REGN-COV2 & Phase $1 / 2$ & NCT04425629 & $\begin{array}{l}2,104 \text { ambulatory patients with } \\
\text { COVID-19 }\end{array}$ \\
\hline AbCellera/Eli Lilly/NIH & $\begin{array}{l}\text { LY3819253 or LY3819253 + } \\
\text { LY3832479 (human IgG1 mAbs } \\
\text { targeting S protein epitope) }\end{array}$ & Phase 3 & NCT04427501 & $\begin{array}{l}800 \text { patients with mild to moderate } \\
\text { COVID-19 }\end{array}$ \\
\hline AbCellera/Eli Lilly/NIH & $\begin{array}{l}\text { LY3819253 or LY3819253 + } \\
\text { LY3832479 }\end{array}$ & Phase 3 & NCT04497987 & $\begin{array}{l}2,400 \text { healthy staff or residents of } \\
\text { skilled nursing facilities }\end{array}$ \\
\hline AbCellera/Eli Lilly/NIH & $\begin{array}{l}\text { LY3819253 versus remdesivir } \\
\text { (small-molecule nucleotide analog } \\
\text { antiviral that blocks viral RNA } \\
\text { polymerase) }\end{array}$ & Phase 3 & NCT04501978 & 10,000 hospitalized patients \\
\hline Vir Biotechnology/GlaxoSmithKline & $\begin{array}{l}\text { VIR-7831/GSK } 4182136 \text { (human IgG1 } \\
\text { mAb targeting S protein epitope) }\end{array}$ & Phase 3 & NCT04545060 & $\begin{array}{l}1,360 \text { non-hospitalized patients with } \\
\text { COVID-19 at high risk }\end{array}$ \\
\hline $\begin{array}{l}\text { BeiGene/Singlomics/Peking } \\
\text { University }\end{array}$ & $\begin{array}{l}\text { BGB-DXP593 (human IgG1 mAb } \\
\text { targeting S protein epitope) }\end{array}$ & Phase 2 & NCT04551898 & $\begin{array}{l}180 \text { patients with mild to moderate } \\
\text { COVID-19 }\end{array}$ \\
\hline $\begin{array}{l}\text { BeiGene/Singlomics/Peking } \\
\text { University }\end{array}$ & BGB-DXP593 & Phase 1 & NCT045332294 & 30 healthy adults $18-60$ years olds \\
\hline $\begin{array}{l}\text { Junshi Biosciences/Institute of } \\
\text { Microbiology, Chinese Academy }\end{array}$ & $\begin{array}{l}\text { JSO16 (human mAb targeting S protein } \\
\text { epitope) }\end{array}$ & Phase 1 & NCT04441918 & 40 healthy participants $15-45$ years old \\
\hline Tychan & TY027 & Phase 1 & NCT04429529 & 32 healthy adults $21-50$ years old \\
\hline Celltrion & $\begin{array}{l}\text { CT-P59 (human mAb targeting } \\
\text { S protein epitope) }\end{array}$ & Phase 1 & NCT04525079 & 32 healthy adults $19-55$ years old \\
\hline $\begin{array}{l}\text { Brii Bio/TSB Therapeutics/Tsinghua } \\
\text { University }\end{array}$ & $\begin{array}{l}\text { BRII-196 (human mAb targeting } \\
\text { S protein epitope) }\end{array}$ & Phase 1 & NCT04479631 & 12 healthy adults $18-49$ years old \\
\hline $\begin{array}{l}\text { Brii Bio/TSB Therapeutics/Tsinghua } \\
\text { University }\end{array}$ & $\begin{array}{l}\text { BRII-198 (human mAb targeting } \\
\text { S protein epitope) }\end{array}$ & Phase 1 & NCT04479644 & 12 healthy adults $18-49$ years old \\
\hline $\begin{array}{l}\text { Sinocelltech/Chinese Academy of } \\
\text { Sciences }\end{array}$ & $\begin{array}{l}\text { SCTA01 (humanized mAb targeting } \\
\text { S protein epitopes) }\end{array}$ & Phase 1 & NCT04483375 & 33 healthy adults \\
\hline Mabwell (Shanghai) Bioscience & $\begin{array}{l}\text { MW33 (human mAb targeting } \\
\text { S protein epitope) }\end{array}$ & Phase 1 & NCT05433048 & 42 healthy adults $18-45$ years old \\
\hline Sorrento/Mount Sinai & $\begin{array}{l}\text { COVI-GUARD/STI-1499 (human mAb } \\
\text { targeting S1 subunit of S protein) }\end{array}$ & Phase 1 & NCT04454398 & $\begin{array}{l}33 \text { hospitalized patients with moderate } \\
\text { COVID-19 }\end{array}$ \\
\hline AstraZeneca/Vanderbilt & $\begin{array}{l}\text { AZD8895 + AZD1061 (IgG1 human } \\
\text { mAbs targeting S protein epitopes) }\end{array}$ & Phase 1 & NCT04507256 & 48 healthy adults $18-55$ years old \\
\hline Hengenix Biotech & $\begin{array}{l}\text { HLX70 (human mAb targeting } \\
\text { S protein epitope) }\end{array}$ & Phase 1 & NCT04561076 & 24 healthy adults $18-60$ years old \\
\hline
\end{tabular}

Source: https://www.antibodysociety.org/covid-19-biologics-tracker/

models ${ }^{1-3}$. Alternatively, chimeric camelid $\mathrm{V}_{\mathrm{HH}} /$ human $\mathrm{Fc}$ nanobodies that were developed for Middle East respiratory syndrome [MERS] also provided passive immunotherapy in a mouse model ${ }^{10}$. Nanobodies are promising because of their small size, stability and potential intracellular uptake and viral neutralization. J.G.: In general, a standard IgG1 is the most established and least risky starting point for an $\mathrm{mAb}$ therapy. Deviations result in increased risk of anti-drug antibody responses that may render the drug ineffective in some recipients. Smaller mAb fragments - Fab fragments, single-chain $\mathrm{Fv}$ fragments and $\mathrm{V}_{\mathrm{HH}}$ nanobodies - have somewhat improved tissue penetration and per-mass binding potency, but the benefit of this small advantage must be balanced against a greatly reduced half-life: these smaller fragments have half-lives on the order of hours, whereas full IgGs' are on the order of weeks.

That said, engineering of the Fc can improve safety by removing the risk both of ADE [antibody-dependent enhancement] (where Fc-receptor bearing immune cells are infected by the virus) and of Fc-receptor-mediated cytokine release by macrophages, can avoid effector-function-mediated off-target or on-target tissue damage, can improve 
half-life (via FcRn [neonatal Fc receptor] half-life extension), and can adapt antibodies for compatibility with bacterial or fungal manufacturing facilities to increase drug scaling. We have chosen to enhance the safety, half-life and capacity for mass production of our antibodies to increase production and reduce cost of the therapy.

\section{What types of Fc-mediated functions of a therapeutic mAb might be important in COVID-19?}

E.O.S.: Fc activities looks to be very important for clearance. There are good studies on this from survivor sera and vaccine sera from Galit Alter ${ }^{11,12}$ and Jeff Ravetch. We are analyzing the $\mathrm{mAb}$ therapeutics for the array of different functions each elicits to map which are key for protection, whether we get certain functions at certain binding sites, etc. This is a major area of discovery.

D.B.: The importance of Fc-mediated effector functions varies greatly for different viruses and different antibodies against the same virus. Blanket rules do not apply. Most judicious for first-in-humans is probably to go with non-engineered human IgG1 Fc (half-life extension excepted).

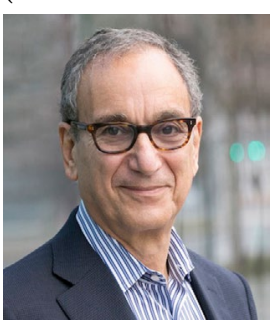

George Scangos, Vir Biotechnology.

\section{George Scangos:}

The capacity of the Fc portion of an $\mathrm{mAb}$ to engage the multiple different human activating or inhibitory Fc receptors ( $\mathrm{FcRs}$ ) could be important to elicit full effectiveness in either therapy or prophylaxis. Through effector functions,

mAbs have been shown to recruit cells of the immune system that attack and kill infected cells, in addition to neutralizing free viruses that spread between cells. The interactions of an antiviral mAb with these FcRs can be assessed in cultured cell by their elicitation of intracellular signals, antibody-dependent cellular cytotoxicity (ADCC),

antibody-dependent cellular phagocytosis or other responses. Different activities come from interactions with different FcRs.

Many mAbs specific for the SARS-CoV-2 $S$ protein, even if potently neutralizing, lack substantial effector function. There may be many reasons for this lack, including the geometry of $\mathrm{mAb}$ binding to the viral $\mathrm{S}$ protein and the number of mAbs that can simultaneously occupy a single $S$ protein. To select $m A$ bs with effector function, it is important to use primary human immune cells to verify activities. Data from these cell-line-based assays can be used to screen for effector function to help select the best candidate to be potentially effective in people. It is also possible to engineer the Fc portion of a mAb to potentially extend the circulation time of the antibody to both provide longer protection and enhancement of the amount of antibody that reaches mucosal surfaces. Vir's lead $\mathrm{mAb}$ for COVID-19 has been designed with the goal of having effector function, extended half-life and increased mucosal penetration. Ongoing clinical trials of this antibody, versus other third-party antibodies that do not contain these properties, will inform how important effector function is for $\mathrm{mAb}$ therapy for COVID-19.

G.G.: A recent study has suggested that RBD-directed antibodies from severely ill COVID-19 patients are more likely to have a certain glycosylation profile (namely, reduced fucosylation) that leads to higher affinity for the Fcy RIII receptors, which in turn correlates with more potent effector functions. Small-scale studies reporting no clinical differences in patients with high-affinity polymorphisms for Fcy receptors suggest that antibody engineering to enhance effector functions is not warranted and may in fact be counterproductive. J.G.: Effector functions have risks. In dengue, removing effector functions from $m A b s$ was shown to avoid $\mathrm{ADE}$ and was therefore desirable to remove. In HIV, effector functions aided in targeting infected cells and were therefore desirable to keep. In influenza, it appears that effector functions aid in clearance of virus, even for antibodies that are not neutralizing, although there is also some evidence suggesting $\mathrm{ADE}$ in influenza. With the novel coronavirus, it depends to what degree antibodies induce ADE. Other coronaviruses have shown ADE. The widespread infection of human cell types, including neurons, causes some concern of effector-function mediated cell clearance. We have chosen to remove effector functions in our antibody therapy to enhance safety, but other groups have chosen to retain effector functions to promote clearance, and they may be correct. Ultimately, this will be resolved in clinical studies.

T.G.: The importance of Fc effector

functions varies dramatically across different viral infections and even across different antibodies to the same virus. The types of Fc-mediated functions that are important for maximal clearance of SARS-CoV-2 are not yet understood and may vary depending on the antibody or combinations of antibodies.

It is well-known that ADCC activity is modulated by the $\mathrm{N}$-linked glycosylation in the $\mathrm{Fc}$ region, and various modifications to the Fc glycan can be engineered to either enhance or diminish this activity.
For example, afucosylated antibodies show increased ADCC activity. In the case of SARS-CoV-2, it is not yet known whether Fc-mediated effector functions are important for protection against disease and, if so, whether enhancement of these activities would be beneficial or potentially harmful.

\section{What are the optimal in vitro systems and models to test activity of leads?}

L.J.S.: Human organoid models for the upper and lower respiratory tract and for the intestine are attractive because they maintain the tissue three-dimensional structures and have multiple interacting cell types. Also, human intestinal organoids have been shown to be susceptible to infection with SARS-CoV-2 ${ }^{13}$.

E.O.S.: We don't yet know what's optimal. It seems like it would be ideal to work with human respiratory cells, but they can clump and not behave well in tissue culture compared with often-used laboratory cell lines like Vero cells. You might get cleaner data using Veros, but you do have to do some additional work to understand relevance to the human tissue.

T.G.: This is not yet known. We need to establish a clear correlation between specific in vitro neutralization assays and in vivo protection - that will tell us what assays are the most predictive. The other problem is that many of the currently used assays are variable amongst each other and even within the same assay performed at different labs. The absence of a uniform, reproducible assay has made the comparison of antibodies from different sources difficult.

\section{What are the preferred ways of measuring neutralization of SARS-CoV-2 in vitro and in vivo?}

L.J.S.: To perform virus neutralization tests, SARS-CoV-2 S pseudotype viruses are used in Biosafety Level (BSL) 2 labs as an alternative to the use of authentic SARS-CoV-2, which requires BSL-3 labs. However, the $S$ pseudotype viruses appear to be more sensitive to virus neutralization by $m A b s$ in several studies versus the authentic SARS-CoV-2, so confirmatory studies in BSL-3 labs are still needed.

There are advantages and disadvantages to each of the SARS-CoV-2 animal models for the testing of human mAbs. The hamster model may best reflect several aspects of COVID-19 infection in humans, but severe fatal disease is not observed. Likewise, there are minimal clinical signs in NHPs and the disease is milder than that in severely affected humans. Also, neither animal model reflects the highest risk groups: the elderly and those with certain co-morbidities. In the 
context of testing $\mathrm{mAb}$ protection, there are differences in effector cells and Fc receptors between humans and the animal models that may influence the outcomes.

E.O.S.: Several in vitro assays are being used, including pseudovirus neutralization tests (putting SARS-CoV-2 S protein onto vesicular stomatitis virus (VSV) or a lentiviral vector); authentic SARS-CoV-2, measured by plaque assay; SARS-CoV-2 engineered to express a Neon green marker, which allows more high-throughput measures or recombinant RBD as a surrogate for neutralization; and cell-cell fusion.

My lab is using VSV pseudovirus, S-protein binding, RBD binding, and cellcell fusion assays. The CoVIC consortium is running all the therapeutic candidates through VSV pseudovirus and SARS-CoV-2 with neon green at BSL-3.

Several in vivo models are in use: mice transgenic for hACE2 receptor (these have been made in different ways by placing the $A C E 2$ gene under different promoters, which can result in somewhat different levels and distributions of hACE2 throughout the mouse), mice infected with adenovirus expressing hACE2, mice infected with a mouse-adapted SARS-CoV-2, Syrian golden hamsters, cats, NHPs and other animals.

CoVIC is examining mice transgenic for hACE2, mice transgenic for hACE2 and human neonatal $\mathrm{Fc}$ receptor ( $\mathrm{FCRn}$ ), and Syrian golden hamsters, followed by NHPs. We will be doing extensive comparison among multiple in vitro assays, in vivo models and human clinical data to try to understand the predictive value of each assay or model.

For most diseases, we evaluate things in a pipeline of in vitro assays, then in rodents, and then in larger animals. Each step is a funnel of selection: success in mice, for example, is often a prerequisite for any further progress to humans. Usually, a final $\mathrm{mAb}$ therapeutic must be able to pass through each of these downselection funnels before any human data are collected on it. So, there is the formal possibility that, in the past, there could have been a therapeutic that would have worked in humans but not in mice. If that's so, we would not have known, as it would have been dropped from further consideration after failure in mice.

This year, for this virus, we will have human clinical data earlier in the process, so we will better understand the predictive value of the animal models for success in humans. The goal of the parallel CoVIC study is to understand, across a large array of antibodies of different epitopes, affinities, etc., what the different in vitro assays are telling us, which combination of antibody attributes we need for protection, which animal models best predict this protection, what types of antibodies fall through or don't fall through the selection criteria we have previously applied, and whether these previous criteria have been the right criteria to apply.

\section{What preclinical studies are needed to fill gaps in our knowledge of SARS-CoV-2 antibody responses?}

L.J.S.: Longitudinal studies of the onset and duration of virus shedding and antibody responses in asymptomatic, mildly and severely affected patients are needed. Also does the magnitude of serum or mucosal neutralizing $\mathrm{Ab}$ response correlate with cessation of virus nasal or fecal shedding or viral load in bronchoalveolar lavage and the resolution of disease? What are the correlates of protection to natural infection? Is there any evidence for $\mathrm{ADE}$ in reinfected individuals who have waning serum antibody levels, although such symptomatic reinfections seem to be rare, and could a similar scenario occur in seropositive vaccinees? What are the safety and efficacy of convalescent plasma treatments in well-controlled clinical trials?

D.B.: We need more information on the role of antibody effector function in anti-SARS-CoV-2 activity and the influence of pre-existing immunity against endemic coronaviruses on antibody responses to SARS-CoV-2.

E.O.S.: There are many gaps. We need to understand whether in vitro neutralization assays adequately recapitulate all viral activities in all cell types in a living thing. Which antibodies work best together - are there synergistic pairs? What is the ideal collection of therapeutic products that we need to have at the ready - do we need to combat viral escape at different sites using different antibody binding footprints, for example? What is the role of recruiting Fc-mediated immune functions in clearance and protection - it looks important, but which functions at what sites? Do we want high levels of some Fc activities or moderate levels? Are there any immune functions we don't want? Do the types of Fc function elicited differ by epitope recognized? Should we be concerned about ADE in vivo? What is the extent to which animal models recapitulate human disease, human protection and human pharmacokinetics? What is the ideal antibody dosage or the minimum that can achieve protection? Which antibodies are potent enough, stable enough and simple enough to manufacture that they can be delivered broadly and affordably around the globe? The consortium I am directing, CoVIC, has set out to answer several of these questions using a library of the current therapeutic candidates and $>100$ other therapeutic mAbs from academics, non-profits and small and large biotechs around the world. J.G.: We don't know what a protective level of antibody is, in a recovered patient, in a vaccinated subject, or in a subject receiving a recombinant antibody. This is a problem. It's not a unique problem - the influenza community cannot agree on what neutralization titer correlates with protection from infection (they use an hemagglutinin inhibition assay instead). However, it is a question that will require a policy to establish some semblance of order in 2021 as people who have been vaccinated, been infected, or received an antibody therapy collectively ask, "Am I protected?" T.G.: Some important gaps in our knowledge include: first, the potential role of pre-existing immunity to endemic coronaviruses in shaping the antibody response to SARS-CoV-2; second, the role of $\mathrm{Fc}$ effector function in protection against SARS-CoV-2 disease in animal models and humans, and third, the longevity of protective neutralizing antibody titers following natural infection and vaccination.

What are the likely optimal scenarios for therapeutic and prophylactic intervention with anti-SARS-CoV-2 mAbs?

L.J.S.: For therapeutic treatment, like for other existing antiviral mAbs (for example, those targeting Ebola virus), it appears essential to treat COVID-19 patients early in the course of the disease. Notably, in the Ebola virus antibody field trials, mAb efficacy was highest in reducing mortality in patients treated early after symptom onset and who had low viral loads and low disease severity biomarkers ${ }^{14}$. Of interest was the finding that the same mAbs tested in NHP models (including the ineffective ZMapp antibody [EB3 cocktail comprising three murine mAbs targeting Ebola virus mucin-like domain, 6D31 and core epitopes of glycoprotein 1]) administered 5 days after Ebola virus challenge showed 100\% survival rates in the NHPs compared with the $34-50 \%$ mortality in the mAb-treated Ebola patients, raising concerns about the predictiveness of the animal models for human responses to $\mathrm{mAb}$ therapy. For SARS-CoV-2, anti-S protein human $\mathrm{mAb}$ treatment injected intraperitoneally into a mouse model had positive outcomes when administered 12 hours after viral challenge ${ }^{2}$. However, like the Ebola mAb treatments, it is less likely that [virally targeted] mAbs will be effective late in the course of COVID-19 disease if viral replication is already decreasing and the ensuing disease 


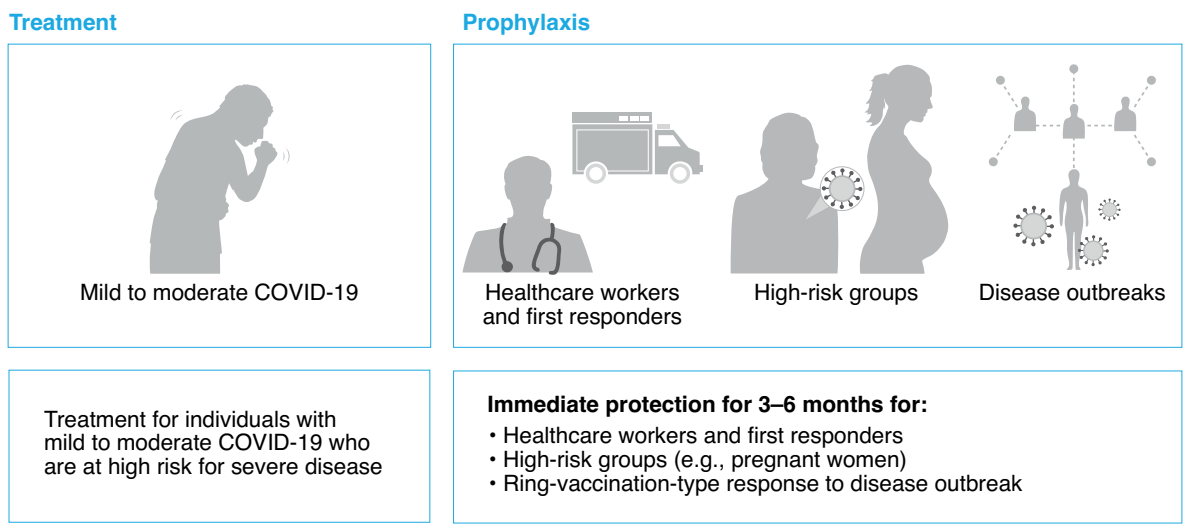

Use cases for prophylaxis with monoclonal antibodies against SARS-CoV-2. Source: Sharon Schendel, La Jolla Institute for Immunology.

course is complicated by host response parameters, such as immune dysregulation, immunopathology and irreversible organ failures.

For prophylactic treatment, the mouse, hamster and NHP model data for SARS-CoV-2 indicate that ideally the mAb would be administered intravenously or via aerosol (to block or reduce lung infection) or intranasally (to block or reduce upper respiratory tract infection) within 12-72 hours pre-exposure to prevent infection or decrease disease $\mathrm{e}^{1-3}$. Instead of a protein $\mathrm{mAb}$, a recent strategy has been to deliver an mRNA encoding an $\mathrm{RSV} \mathrm{mAb}$ (palinozumab) to the lung via intratracheal aerosols ${ }^{15}$.

G.G.: The data from convalescent plasma therapy argue that $\mathrm{mAbs}$ are likely to be beneficial in the management of severe COVID-19 disease. Passive immunization with $\mathrm{mAbs}$ will almost certainly require the use of Fc-engineered antibodies having amino acid substitutions that greatly prolong the persistence of antibodies in circulation from $\sim 21$ days to $\sim 85$ days. Two sets of Fc mutations conferring long half-life (so called "YTE" and "LS") have been validated clinically, and even though both have certain liabilities in terms of reduced effector functions and bioprocessing characteristics, they are nonetheless attractive for COVID-19 mAbs. In fact, at least two SARS-CoV-2 neutralizing antibodies in clinical trials have been engineered for long half-life. If all goes well, then the prospect of having a prophylactic antibody that confers protection for over 6 months after a single intravenous administration may be within reach.

J.G.: We have learned from the small-molecule drug [Veklury] remdesivir and convalescent plasma studies that antivirals are more effective the earlier that they are applied to the patient. This is consistent with observations we have had with other viruses, like Ebola. Later stage COVID-19 disease still benefits from antibody therapy but is complicated by tissue damage, widespread virus in tissues of the body, and an excessive and self-destructive immune response, which requires steroids, other anti-inflammatory agents, anticoagulation agents, oxygen and other support beyond direct viral targeting. For these reasons, we need two types of medicines for people to take as soon as they have a SARS-CoV-2 positive RNA test result: a high-dose intravenous treatment for patients admitted to hospitals, and a post-exposure and early-exposure prophylactic for people who have just started to have symptoms or don't even yet have symptoms and don't want to risk getting sick or infecting others. The latter is the approach that has eliminated rabies from the human experience, and should be applied to COVID-19.

The convenience and cost of such a prophylactic will determine how early people would take the medicine. We have performed thermostabilization engineering of our antibody therapeutic to tolerate high-concentration delivery in a subcutaneous injection and high-efficiency manufacturing to produce large numbers of doses inexpensively. A subcutaneous injection that prevents COVID-19 could be performed as an outpatient procedure in clinics, retirement centers, cruise ships and homes.

\section{What considerations are important in determining the optimal half-life of a SARS-CoV-2 mAb?}

E.O.S.: If an antibody is used therapeutically (after exposure), for a non-chronic infection, you don't need an antibody to last as long - many days to a couple of weeks. If an antibody is used for prophylactic (preventative) use, it may need to last longer. You don't know when the person will be exposed - the antibody needs to stay around long enough at high enough concentrations to be protective when its recipient is exposed.

T.G.: For prophylaxis, a long serum half-life is optimal to allow prolonged protection without repeat antibody administration. This can be achieved using various half-life extension mutations, which prolong the serum half-life of IgG antibodies by about three- to fourfold. Based on precedent set with other viral infections, prophylactic protection for up to 6 months following a moderate intramuscular or subcutaneous dose (for example, 100-300 milligrams) of a potent SARS-CoV-2 neutralizing antibody is likely achievable. In the case of therapy, the half-life of the antibody is less relevant given the shorter duration required for therapeutic action. However, certain half-life extension modifications are also associated with increased tissue concentrations, particularly at mucosal surfaces, which could provide an efficacy advantage in both the prevention and treatment settings.

D.B.: For prophylaxis, long half-life via half-life extension mutations would seem optimal. For therapy, it could be more complicated. If (and it is a big if) any ADE effects are associated with SARS-CoV-2 mAbs, then a long half-life might be disadvantage.

How do antibody development programs minimize the emergence of viral escape mutants?

L.J.S.: The best approach based on the SARS-CoV-2 literature appears to be a cocktail of neutralizing mAbs to non-competing epitopes. This is important not only to improve the efficacy, but also to prevent SARS-CoV-2 escape mutants that are more likely to arise based on single mAb therapy ${ }^{16}$.

D.B.: Selection of antibodies that bind to conserved features of, for example, the $\mathrm{S}$ protein of different strains of SARS-CoV-2, and even different coronaviruses, and neutralize those coronaviruses would seem to provide a good opportunity to minimize neutralization escape by SARS-CoV-2.

Generally, pan-virus family antibodies are attractive because they are probably more difficult to escape from for any given virus, and they may even help protect against newly emerging viruses of the family (for example, SARS-CoV-3?).

J.G.: Although SARS-CoV-2 virus exhibits a slow mutation rate relative to influenza, some in vitro studies have suggested that escape mutants could emerge once confronted with $\mathrm{mAb}$ therapeutics in vivo. Broadly neutralizing antibodies can be 
selected for that preferentially recognize conserved positions of viral coat proteins and therefore are harder to escape by the virus. Alternatively, cocktails of multiple antibodies could be used to inhibit escape mutants, similarly to HIV multidrug cocktails, provided that all components of the cocktail are independently neutralizing. A creative solution to cocktails can also be found in the marketplace: with multiple antibody therapeutics reaching the market, it becomes possible to externalize the challenge of generating a cocktail to the doctors: patients could be first given an inexpensive monoclonal drug $\mathrm{A}$, and rare escape events could be followed up with cocktail drug B.

T.G.: Antibodies that bind to highly conserved epitopes (that is, broadly neutralizing antibodies) are likely to be more refractory to escape by SARS-CoV-2 due to interactions that have been conserved in nature and thus are likely important for viral fitness.

\section{Are cocktails of mAbs likely to be preferable to antibody monotherapy for SARS-CoV-2?}

T.G.: Not necessarily. SARS-CoV-2 is less genetically diverse than most other RNA viruses, which may translate into a reduced ability to escape from neutralizing antibodies, especially broadly neutralizing antibodies that target conserved residues important for viral fitness. The RSV mAb Synagis has been successfully used for decades without the emergence of clinically significant resistance, and more recently, a single anti-Ebola $\mathrm{mAb}(\mathrm{mAb} 114)$ showed comparable therapeutic efficacy to ZMapp's three-mAb cocktail (EB3) in humans with established Ebola virus disease.

D.B.: Controversial. Some argue to go into humans with cocktails from the get-go; others argue to try monotherapy under controlled conditions and if neutralization escape is observed then be ready with further mAbs. In any case, given capacity limitations on $\mathrm{mAb}$ production and the unknowns of dealing with a new virus, it would seem prudent to bring several or many $\mathrm{mAbs}$ to the clinic.

G.G.: Antibody cocktails seem to provide a synergistic effect in terms of in vitro neutralization and are advantageous in reducing the possibility of selection of escape mutants in patients. As Regeneron's Ebola $\mathrm{mAb}$ cocktail has demonstrated, the added complexity in bioprocessing and quality control in using mixtures of mAbs can be managed.

G.S.: The ultimate goal is to develop a highly safe and effective product that can reach the most patients and save the most lives. Achieving this goal with a single $\mathrm{mAb}$ has the advantage, over cocktails of mAbs, of enabling the production of more doses and thus the ability to reach more patients, making the most of existing global manufacturing capacity.

The global sequencing of SARS-CoV-2 isolates clearly demonstrates that the virus is mutating. A successful product must provide a high barrier to resistance and remain effective as new variants arise. One approach to this is to is to use a cocktail. However, if multiple drugs used in a cocktail have a relatively low barrier to resistance, as is seen for HIV and HCV, patients develop multidrug resistance. Thus, developing an antibody with high barrier to resistance could be even more effective than a cocktail of antibodies to which resistance emerges rapidly to the individual components.

Ebola is an example illustrating how the adage "more is better" is not always true - Zmapp's antibody cocktail didn't show the efficacy that single-antibody treatment, mAb114, demonstrated. (mAb114 was identified by Vir scientists working with the US National Institutes of Health (NIH) and others.) In clinical studies, this single antibody was shown to significantly decrease mortality to the same extent as a cocktail of three antibodies from Regeneron.

Vir's lead antibody for COVID-19, VIR-7831, was designed to be an effective monotherapy and is currently being tested in a phase 3 trial. This antibody targets a highly conserved epitope that is shared between a large array of coronaviruses, is highly potent at blocking infection in vitro, and has the ability to recruit immune cells to kill infected cells. It also has been designed to provide up to six months of potential protection. In a global pandemic, where demand is outpacing supply, the difference between a single well-designed, highly potent $\mathrm{mAb}$ and a cocktail of multiple antibodies could be millions of lives. J.G.: Antibody cocktails provide two benefits and one challenge. First, cocktails make it harder for an escape mutant to appear in the subject, as an effective escape mutant would need to escape both antibodies in the cocktail. Second, cocktails can boost performance of two or three relatively less potent antibodies as they synergize. Historically, synergy can very helpful for the first wave antibody discovery, where there hasn't been as much time to optimize individual antibodies (although all antibodies in all lead candidate cocktails appear excellent).

The challenge of a cocktail is the additional cost in GMP [good manufacturing practice] certification, complexity in manufacturing, and efforts of formulation. Such additional complexities could influence the final cost of medicine.

\section{Is antibody-dependent enhancement a concern, and what kind of testing is needed to give confidence it will not be an issue for anti-SARS-CoV-2 mAbs?}

L.J.S.: For coronaviruses, as exemplified by feline coronaviruses, ADE disease is associated with Fc-mediated entry into Fc-receptor-bearing cells (macrophages for feline coronavirus). A major issue is that most experimental feline coronavirus vaccines trigger $\mathrm{ADE}$, which causes a more severe disease in immunized cats than in control cats after virus challenge.

An alternative mechanism for $\mathrm{ADE}$ has been described for MERS-CoV, whereby neutralizing antibodies bind to the S-protein, triggering a conformational change of the spike and mediating viral entry into Fc-receptor-expressing cells through canonical viral-receptor-dependent pathways $^{17}$. For SARS-CoV-2 mAbs, if $\mathrm{ADE}$ of disease is evident, this may necessitate reducing $\mathrm{Fc}$ receptor binding to minimize the possibility that subtherapeutic viral-neutralizing $\mathrm{mAb}$ could promote ADE. However, in the animal models currently tested and in people with COVID19 given convalescent plasma, there is no reported evidence for $\mathrm{ADE}$ of disease. D.B.: Even for dengue virus, the classic example quoted for $\mathrm{ADE}$ of human disease, it only happens under very specific conditions. No ADE has been described for 20 years of Synagis use in humans. The best approach is to monitor antibody levels and disease courses carefully to maximize chances of detecting any adverse effects. G.G.: Although earlier reports on adverse effects of antibodies in SARS patients and in vitro data with SARS-CoV-2 had initially raised concerns about $\mathrm{ADE}$ disease and/or enhanced respiratory distress ${ }^{18}$, I think that the data pointing to the safety of plasma therapy and also the findings of more recent preclinical studies are much more reassuring.

G.S.: Antiviral antibodies have been given with no evidence of ADE for more than 100 years, and of course babies are born with their mother's antibodies. Anti-SARS-CoV-2 $\mathrm{mAbs}$ are not anticipated to be different, and the absence of safety signals in the 70,000 patients with COVID-19 given convalescent plasma strongly supports this expectation. Nevertheless, robust clinical trials to document their safety are certainly needed.

The biology of ADE has been observed in dengue when, rarely, cross-reactive antibodies from a previous infection help 
the virus enter macrophages. However, dengue can produce infectious virus particles in these cells, but the evidence is that SARS-CoV-2 cannot. Like most viruses, antibody-dependent uptake of SARS-CoV-2 by macrophages degrades the virus. This antibody 'effector function' is a normal mechanism of protection rather than enhancement. This complex area of science has been reviewed recently in several publications (for example, ref. ${ }^{19}$ ).

J.G.: Although all current phase 2 studies of $\mathrm{mAb}$ therapies are showing benefit in COVID-19, in our therapeutic program, we have chosen the risk mitigation strategy of using a well-established Fc-engineering approach to remove effector functions. We made this choice for four main reasons. First, classic ADE, in which antibodies expand infected cell classes by facilitating uptake of viral particles by Fc-receptor-bearing immune cells, has resulted in failed clinical programs for dengue and RSV, and ADE has been observed for related coronaviruses including MERS, SARS and common cold coronaviruses. Second, effector functions could exacerbate cytokine release, potentially contributing to inflammation and immune-mediated tissue damage. Third, effector functions cause antibodies to attach to and mark infected cells for destruction by immune cells. While this is valuable in treating HIV, where infected tissues are largely $\mathrm{T}$ cells, it could pose health hazards against SARS-CoV-2, where the virus can infect the brain, the heart and other sensitive organs. Finally, in the event that the antibody has some cross-reactivity to any human tissue, effector functions could cause those tissues to be attacked. Ultimately, clinical studies will determine whether the removal of effector functions resulted in any improvement in safety and efficacy of antibody therapies.

T.G.: Although ADE of infection has frequently been observed in vitro for many viruses, $\mathrm{ADE}$ of viral disease in humans has only been described clinically in (1) children given formalin-inactivated RSV or measles vaccines and (2) dengue hemorrhagic fever following secondary infection with a heterologous dengue serotype. In the RSV and measles cases, ADE of disease was associated with the induction of non-neutralizing antibodies that formed immune complexes with the virus. This mechanism of ADE is unlikely to occur in the setting of prophylaxis or therapy with highly potent neutralizing monoclonal antibodies to SARS-CoV-2 but may be of concern in the setting of convalescent plasma therapy or vaccination due to the high variability in plasma neutralizing
Partially open SARS-CoV-2 S trimer

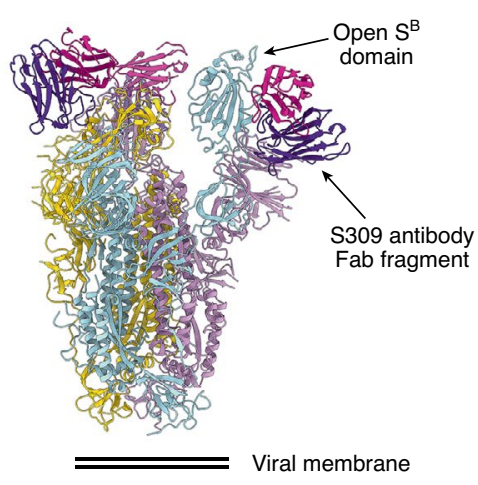

Structure of the SARS-CoV-2 S glycoprotein in complex with the S309 neutralizing mAb Fab fragment. Reprinted with permission from ref. ${ }^{20}$, Springer Nature.

titers and heterogeneity of response to vaccines. In the case of dengue, $\mathrm{ADE}$ can occur when serum antibody concentrations are within a specific subneutralizing range at the time of secondary infection with a heterologous dengue serotype. The mechanism of enhanced infection involves uptake of virus-immune complexes by Fcy receptors expressed on target immune cells. Because coexpression of ACE2 and Fc $\gamma$ Rs on target cells is limited, this mechanism of ADE is unlikely to occur in the context of SARS-CoV-2.

\section{Which antiviral mAb programs have clinical data that you are most excited about, and why?}

L.J.S.: Two current mAb programs are advanced based on their published data: one is developed by a team headed by Jim Crowe at the Vanderbilt Vaccine Center, the other by Regeneron Pharmaceuticals. These groups have developed cocktails of virus-neutralizing $\mathrm{mAbs}$ to non-competing epitopes on SARS-CoV-2 S protein that have shown efficacy therapeutically or prophylactically in hamster, mouse or NHP animal models ${ }^{2,3}$.

G.G.: It is too early to tell which clinical $\mathrm{mAb}$ programs have a higher probability of success. One complicating factor in the design and evaluation of clinical trials for evaluating mAbs for therapy is that most patients having severe disease are likely to be older and have multiple comorbidities. These factors may affect $\mathrm{mAb}$ biodistribution and interactions with the innate immune system.

J.G.: The first round of $\mathrm{mAb}$ therapeutics to enter phase 2 (by Regeneron, AbCellera/ Lilly and Vir/GlaxoSmithKline) are providing valuable support to the general hypothesis that antibody therapy can provide therapeutic and prophylactic benefit, as well as provide specific guidance around dosages and study sizes sufficient appropriate for rapid approval of additional $\mathrm{mAb}$ therapies to the marketplace. Slightly different decisions with respect to effector functions, half-life extension, and cocktail versus $\mathrm{mAb}$ monotherapy will be valuable to track as they play out in this first generation of therapies.

T.G: The programs from both Lilly and Regeneron have demonstrated exceptional safety, even at very high doses. In the absence of real efficacy data for vaccines, safety is going to be a big driver, and antibodies have so far passed that test with flying colors. I am also excited about the demonstrated impact on viral loads and hospitalization rates, although more work will have to be done to fully understand the benefit. Of all drug classes, mAbs have clearly demonstrated to be the most efficacious to date.

\section{Who are the optimal target popula- tions for phase 3 trials of antiviral mAbs for COVID-19?}

D.B.: For prophylaxis, a good target population to test $\mathrm{mAb}$ efficacy would seem to be households or groups living together in which one member is infected (since transmission is known to be high in that environment) and other members(s) are at high risk (so protection from severe disease as well as from infection can be assessed). For therapy, efficacy is most likely if mAbs are given early. For late-stage infection, one might think of combining antiviral mAbs with anti-inflammatory mAbs and/or antiviral drugs.

J.G.: It is critical that typical clinical study exclusion criteria should be relaxed here, as a large proportion of those who need the medicine most (elderly and those with pre-existing medical conditions) may otherwise be excluded from studies. If exclusion criteria are applied, a separate cohort that contains the excluded subjects should be considered.

T.G.: In the setting of prophylaxis, mAbs could potentially be used alone or as a complement to vaccines. A potent $\mathrm{mAb}$ with half-life extension enabling safe and reliable protection for up 12 months could be used as a vaccine alternative for individuals who respond poorly to, are not eligible for, or are simply unwilling undergo vaccination. Antibodies could also be used as additional protection for individuals at particularly high risk of developing complications of COVID-19, such as the elderly and individuals with certain comorbidities. Finally, unlike vaccines, 
mAbs have potential utility in outbreak and postexposure prophylaxis settings, with the ease of intramuscular or subcutaneous administration likely preferred in these circumstances. Based on the broad potential uses of mAbs in prophylaxis, phase 3 trials should ideally include individuals at high risk of exposure to SARS-CoV-2, either due to a documented known exposure or ongoing risk based on occupation or housing situation, as well as individuals at high risk of complications of COVID-19 should they develop SARS-CoV-2 infection.

In the early-infection setting, individuals most likely to benefit from $\mathrm{mAb}$ therapy include the elderly, the immunocompromised and others at highest risk of development of complications from COVID-19. The goals of such therapy are to reduce hospitalizations, intensive care unit admissions and deaths, as well as to reduce the duration of disease. In the outpatient setting, the ability to deliver this therapy via intramuscular or subcutaneous injection is likely to be preferred by patients and providers alike.

In the setting of late, serious infections, $\mathrm{mAbs}$ are likely to be less effective than in prophylaxis or early infection. Much of the pathology of late-stage disease may be due to the host immune response combined with exacerbation of underlying comorbid conditions, rather than ongoing viral replication. Combination therapy consisting of antiviral mAbs and agents designed to address immune dysregulation may be required for these complex cases.

What issues are important to consider for large-scale manufacture of mAbs?

J.G.: US National Institute of Allergy and Infectious Disease (NIAID) Director Anthony Fauci estimates that the United States will require 40 million doses of a COVID-19 antibody therapy, and an analysis of hospitalizations suggests that over 2.5 million doses per month would be likely required globally for the urgent care setting alone. Even with 30,000-liter reactors operating continuously at multiple facilities, there will be a problem of supply. In addition to production, there are problems of national availability. An excessive focus on US supply could leave many areas of the world unprotected, as appears to be the case already with vaccines. International partnerships for manufacturing and distribution, as well as exploration of other manufacturing platforms, will be required to address this global need.

At Centivax, to maximize the manufacture of our Centi-B9 (a monoclonal IgG1 with LALA and LS mutations and thermostability optimization to enable better expression and concentration for subcutaneous injection delivery), we optimized yield in mammalian (at ATUM and Millipore), bacteria (at SwiftScale), and fungal expression systems. Although some of these systems require additional INDs [Investigational New Drug applications], a multiplatform manufacturing strategy enables a dramatic increase in the number of doses that can be manufactured, and therefore a decrease in the cost per dose that can be offered to global markets. The different expression systems offer a balance of established $\mathrm{CHO}$ production versus potential advantages of the newer but less established platforms (availability of capacity, speed, cost of goods, and scale).

Prefilled fixed-dose syringes are convenient for distribution and can be administered outside of a hospital setting, but are limited in application to postexposure prophylaxis and early-stage disease due to their lower dose. Bioengineering methods can be used to optimize antibodies to better tolerate the high concentrations necessary for effective subcutaneous injection. In general, it is my hope that the pandemic will usher in a renaissance of biologics manufacturing technologies to the general benefit of all medicines made thereafter.

Moderna's ability to complete GMP of their RNA vaccine in 43 days was a powerful demonstration of the advantages of genetic delivery platforms. Traditional antibody manufacturing routinely can take 18 months. Even during a pandemic, manufacturing is likely to be one of the central bottlenecks in bringing antibody therapeutics to market against the novel coronavirus. Being able to deliver RNA or DNA and having the patient act as their own bioreactor avoids a substantial amount of time and complexity involved in traditional protein drug development. Compared with protein mAb products, RNA and DNA may have advantages with respect to manufacturing and delivery. The challenge is in generating a high enough titer in vivo to provide protection. A proof of concept published by Moderna ${ }^{21}$ included RNA-delivered Chikungunya antibodies that achieved doses approaching $20 \mu \mathrm{g} \mathrm{mL}^{-1}$, suggesting that this could be a viable means of delivering anti-SARS-CoV-2 antibodies. G.G.: In response to the urgent need for COVID therapeutics, pre-IND studies of COVID-19 therapeutic mAbs have been accelerated by perhaps threefold or more relative to what had been typical for antibody therapeutics. Manufacturing has to rely on established and fully de-risked technologies - namely, expression in $\mathrm{CHO}$ cells. Cell-line development, which is typically relatively lengthy, can also be completed much faster than typically done, although at a commercial risk. The fact that the therapeutic dose will be of the order of a gram or more of $\mathrm{mAb}$ (based on extrapolation from what has been reported for NHP challenge models) means that almost surely administration will have to be intravenous. The high dosing may pose additional challenges for formulation and release testing.

G.S.: Over the past 20 years, the productivity of mAb manufacture has undergone dramatic improvements in yields/ productivity and in the worldwide industry convergence in manufacturing engineering and process technology. Today, there are approximately 25 global facilities with very large scale (VLS) stainless steel fermenters. All of these facilities are readily adaptable to the manufacture of any recombinant antibody and are individually capable of manufacturing up to 10 tons of antibodies every year.

The economies of scale in these VLS facilities are critical to delivering both the tens of millions of doses and acceptable cost of goods to patients worldwide. However, only a fraction of this capacity was available at the onset of the COVID19 pandemic. Now, a large portion of this worldwide capacity has been reserved by Vir Biotechnology and other leading companies for the manufacture of anti-COVID- 19 $\mathrm{mAb}$ treatments.

The peak demand for the first COVID-19 mAbs could exceed the largest blockbuster therapeutic mAbs in the oncology and inflammation disease areas by a factor of five or more. To achieve optimal clinical efficacy, the successful COVID-19 mAbs will also need to demonstrate optimal efficiency in manufacturing. This will include the productivity of the cell line, highly efficient upstream and downstream processes and drug product formulations that are stable as liquid solutions at high antibody concentrations. Antibodies that are effective as monotherapies, have extended treatment duration and that are effective at lower clinical dosages could be highly advantaged.

Vir selected its lead mAb (VIR-7831) both with strategic consideration of these clinical characteristics and with the intention that projected manufacturing constraints would not impact our ability to treat the world's patients, if successful.

\section{Any other closing thoughts?}

G.S.: While collaborations between companies and between industry and academia have always fueled therapeutic innovation, the volume, pace and urgency at 
which groups were able to work together to produce potential solutions to SARS-CoV-2 has been unprecedented.

In the case of Vir's partnership with GlaxoSmithKline, the substantial global need brought to bear a partnership that combines the unique scientific and technical expertise of each company to advance better solutions faster. Our joint commitment to moving quickly to make an impact on the pandemic was evident from the beginning. From the initiation of our discussions with GSK to a signed agreement took 18 days. That is unprecedented in my experience and reflects the views of both companies that we have a responsibility to put aside normal considerations and work together quickly to bring potentially life-saving medicines to patients as quickly as possible.

We established a network of partners that span academia, government, other pharma companies and multiple manufacturing partners that have enabled us to accelerate the development of what we believe could be a best-in-class $\mathrm{mAb}$, which is currently being evaluated in a phase 3 trial. In addition to moving rapidly to produce clinical material and to determine the efficacy of the product, this partnership network has accelerated the development of a high-yield commercial process that could provide up to ten million doses in 2021.

If successful, we will have condensed a drug development process that typically takes 8-10 years into just over a year. Now, we need to invest in being better prepared to address emerging pathogens to avoid future pandemics.

J.G.: Centivax is also deeply appreciative of its partners: Galveston National Laboratory, the University of Texas Medical Branch, the US Department of Defense, USAMRIID, the Peter Kim lab at Stanford University, the Nigel Temperton lab at the University of Kent, Sino Biological, Thermo Fisher, ATUM, Millipore and Charles River
Laboratories. All have been remarkable partners in this effort to develop an abundant and affordable therapeutic against COVID-19.

Antibodies historically have been thought of as potent but expensive medicines, often costing more than $\$ 10,000$ per treatment. This is not cost-of-goods: an mAb therapy costs roughly $\$ 100$ per gram to produce, and 1-2 gram doses appear to provide benefit in clinical studies. Subsidization of mass production by governments combined with a high-volume/low-unit-cost pricing model could be used to make sure that all people have access. Governments cannot subsidize $\$ 10,000$ per dose for all sick citizens, but they could subsidize a medicine at $\$ 500$ per dose. Alternative manufacturing platforms could further increase access for nations that may not have GMP capability for $\mathrm{CHO}$, but do have GMP capability for bacterial or fungal expression platforms.

L.J.S.: Development of neutralizing cross-reactive human mAbs to conserved epitopes on SARS, SARS-CoV-2 and SARS-related coronaviruses that can inhibit infection by multiple sarbecoviruses is critical for immediate availability of immunotherapies for future emerging SARS-related coronavirus outbreaks. Identification of such conserved epitopes are also important for the design of broadly reactive vaccines to prevent future SARS-related coronavirus infections. Nanobodies are a promising approach because of their small size, stability and potential for intracellular uptake and intracellular viral neutralization.

D.B.: We absolutely have to stockpile broadly neutralizing mAbs to coronaviruses so we have a better chance of combatting SARS-CoV-3 when it emerges! For the present therapies, there will be many challenges in distributing products to low- and middle-income countries. I recommend reading the recent excellent report "Expanding Access to Monoclonal Antibodies" from the Wellcome Trust and IAVI [the International AIDS Vaccine Initiative].

T.G.: The identification and stockpiling of broadly protective coronavirus mAbs and vaccines should be a priority going forward. We have now seen the emergence of four endemic coronaviruses (HKU1, NL63, OC43 and 229E), two highly pathogenic coronaviruses that caused deadly outbreaks (SARS and MERS), and one coronavirus that caused the current COVID-19 pandemic (SARS-CoV-2). Given that the latter three viruses all emerged over the past two decades, it appears not to be a matter of 'if' but a matter of 'when' the next pathogenic coronavirus will spill over from zoonotic reservoirs into the human population.

\section{Interviewed by Laura DeFrancesco}

Published online: 21 October 2020 https://doi.org/10.1038/s41587-020-0732-8

References

1. Alsoussi, W. B. et al. J. Immunol. https://doi.org/10.4049/ jimmunol.2000583 (2020).

2. Zost, S. J. et al. Nature 584, 443-449 (2020).

3. Rogers, T. F. et al. Science 369, 956-963 (2020).

4. Chi, X. et al. Science https://doi.org/10.1126/science.abc6952 (2020).

5. Vega, C. G. et al. PLoS Pathog. 9, el003334 (2013).

6. Burns, J. W., Siadat-Pajouh, M., Krishnaney, A. A. \& Greenberg, H. B. Science 272, 104-107 (1996).

7. Feng, N. et al. J. Clin. Invest. 109, 1203-1213 (2002).

8. Bidgood, S. R., Tam, J. C., McEwan, W. A., Mallery, D. L. \& James, L. C. Proc. Natl Acad. Sci. USA 111, 13463-13468 (2014).

9. Saif, L. J. Eur. Med. J. https://doi.org/10.33590/emj/200324 (2020).

10. Stalin, R. V. et al. Sci. Adv. 4, eaas9667 (2018).

11. Atyeo, C. et al. Immunity 53, 524-532.e4 (2020).

12. Zohar, T. \& Alter, G. Nat. Rev. Immunol. 20, 392-394 (2020).

13. Lamers, M. M. et al. Science 369, 50-54 (2020).

14. Mulangu, S. et al. N. Engl. J. Med. 381, 2293-2303 (2019).

15. Tiwari, P. M. et al. Nat. Commun. 9, 3999 (2018).

16. Baum, A. et al. Science https://doi.org/10.1126/science.abd0831 (2020).

17. Wan, Y. et al. J. Virol. 94, e02015-e02019 (2020).

18. Liu, L. et al. JCI Insight 4, e123158 (2019).

19. Arvin, A. M. et al. Nature 584, 353-363 (2020)

20. Kose, N. et al. Sci. Immunol. 4, eaaw6647 (2019).

21. Pinto, D. et al. Nature 583, 290-295 (2020). 